\title{
High MICs for Vancomycin and Daptomycin and Complicated Catheter-Related Bloodstream Infections with Methicillin-Sensitive Staphylococcus aureus
}

\author{
Rafael San-Juan, Esther Viedma, Fernando Chaves, Antonio Lalueza, Jesús Fortún, Elena Loza, \\ Miquel Pujol, Carmen Ardanuy, Isabel Morales, Marina de Cueto, Elena Resino-Foz, \\ Alejandra Morales-Cartagena, Alicia Rico, María P. Romero, María Ángeles Orellana, \\ Francisco López-Medrano, Mario Fernández-Ruiz, José María Aguado
}

\begin{abstract}
We investigated the prognostic role of high MICs for antistaphylococcal agents in patients with methicillin-sensitive Staphylococcus aureus catheter-related bloodstream infection (MSSA CRBSI). We prospectively reviewed 83 episodes from 5 centers in Spain during April 2011-June 2014 that had optimized clinical management and analyzed the relationship between E-test MICs for vancomycin, daptomycin, oxacillin, and linezolid and development of complicated bacteremia by using multivariate analysis. Complicated MSSA CRBSI occurred in 26 (31.3\%) patients; MICs for vancomycin and daptomycin were higher in these patients (optimal cutoff values for predictive accuracy $=1.5$ $\mu \mathrm{g} / \mathrm{mL}$ and $0.5 \mu \mathrm{g} / \mathrm{mL}$ ). High MICs for vancomycin (hazard ratio $2.4,95 \% \mathrm{Cl} 1.2-5.5$ ) and daptomycin (hazard ratio $2.4,95 \% \mathrm{Cl} 1.1-5.9$ ) were independent risk factors for development of complicated MSSA CRBSI. Our data suggest that patients with MSSA CRBSI caused by strains that have high MICs for vancomycin or daptomycin are at increased risk for complications.
\end{abstract}

Staphylococcus aureus bacteremia is an issue of con$\mathcal{N}$ cern because of high rates of illness and death for this condition (1). Some studies have identified high MICs for vancomycin (defined as an MIC $\geq 1.5 \mu \mathrm{g} / \mathrm{mL}$ by E-test) as a simple laboratory-based marker, which has been shown

Author affiliations: University Hospital-Research Institute 12 de Octubre, Madrid, Spain (R. San-Juan, E. Viedma, F. Chaves, A. Lalueza, E. Resino-Foz, A. Morales-Cartagena, M.Á. Orellana, F. López-Medrano, M. Fernández-Ruiz, J. María Aguado); Hospital Universitario Ramón y Cajal, Madrid (J. Fortún, E. Loza); University Hospital-Bellvitge Institute for Biomedical Research, Barcelona, Spain (M. Pujol, C. Ardanuy); Hospital Universitario Virgen de la Macarena, Seville, Spain (I. Morales, M. de Cueto); Hospital Universitario La Paz, Madrid (A. Rico, M.P. Romero)

DOI: http://dx.doi.org/10.3201/eid2206.151709 to be associated with a worse prognosis in methicillinresistant $S$. aureus (MRSA) bacteremia in terms of treatment failure (2-4) and increased mortality rates $(5,6)$. Such increased mortality rates associated with a high MIC for vancomycin has also been reported in methicillin-sensitive S. aureus (MSSA) bacteremia $(7,8)$. However, the use of death as a study outcome and the fact that these studies were performed with patients with different sources of bacteremia make it difficult to interpret study results. Such findings could not be confirmed in large cohorts of patients with MRSA (9-11) or MSSA (12) bacteremia from different sources.

To avoid these confounding factors, we focused on a more homogeneous population of patients with MSSA catheter-related bloodstream infection (MSSA CRBSI) for whom specific efforts were devoted to optimize clinical management. In a previous retrospective study, we observed that high MICs for vancomycin were associated with development of complicated MSSA CRBSI, regardless of the initial antimicrobial drug therapy used (vancomycin or $\beta$-lactams), which suggested that intrinsic characteristics of these strains might explain their pathogenic role in development of complicated bacteremia (13). We designed this multicenter prospective study to confirm the prognostic role of high MICs for vancomycin and to explore if increased MICs for other antistaphylococcal agents could also influence the risk for developing complicated MSSA CRBSI.

\section{Materials and Methods}

\section{Study Design and Setting}

We conducted a prospective, observational, multicenter study during April 2011-June 2014 in 5 hospitals in Spain in which we included patients given a diagnosis of MSSA 
CRBSI according to standard a definition (14) that fulfilled 5 criteria: 1 ) age $\geq 18$ years and a life expectancy $\geq 7$ days; 2) no use of daptomycin within the 3 months preceding the incident MSSA CRBSI episode; 3 ) removal of the intravascular catheter suspected to be the source of CRBSI within a maximum of 72 hours from sampling of the first blood cultures yielding MSSA; 4) a maximum of 72 hours of treatment with glycopeptides; and 5) at least the first blood culture isolate available for microbiological analysis. All included patients signed a study-specific informed consent form. The study was approved by the ethics research boards at all participating centers.

\section{Management of MSSA CRBSI}

Although the nature of the study was not interventional, the adherence to evidence-based quality-of-care measures was strongly encouraged among participating investigators to optimize the therapeutic management of MSSA CRBSI. These measures were extraction of follow-up blood cultures, ordering echocardiography or venous Doppler ultrasound examination for patients with clinical indication, early use of intravenous cloxacillin or other highly effective antimicrobial drugs for treatment of for MSSA as definitive therapy, and adjustment of treatment duration according to the complexity of the infection.

\section{Definitions}

Recorded clinical variables were age; site of acquisition of infection; concurrent conditions, as assessed by the Comorbidity Index of Charlson et al. (15); prognosis of the underlying disease (classified according to the McCabe and Jackson modified criteria as rapidly fatal [when death was expected within 3 months], ultimately fatal [when death was expected within a period of $>3$ months but $<5$ years] and nonfatal [when life expectancy was $>5$ years]) (10); type of intravenous catheter; timing of catheter removal; treatment administered; duration of fever; duration of bacteremia; Pitt bacteremia score (17); development of septic shock; endovascular infection or complicated bacteremia (defined below); and all-cause and MSSA CRBSI-attributable mortality rates at 30 days. MSSA CRBSI-attributable deaths at 30 days were identified as per the clinical judgment of the investigator.

Antimicrobial drugs were classified within 1 of the following mutually excluding categories: glycopeptides; daptomycin; antistaphylococcal $\beta$-lactams (parenteral cloxacillin, cefazolin or other active $\beta$-lactams [including parenteral amoxicillin/clavulanate, piperacillin/tazobactam, imipenem, or meropenem]); non- $\beta$-lactam agents with in vitro activity against MSSA (antistaphylococcal quinolones, tigecycline, or linezolid); and noneffective agents. Timing to initiation of antimicrobial drug therapy and duration of treatment were also recorded.
Empiric therapy was defined as therapy administered before identification and antimicrobial susceptibility testing of the isolate. Complicated bacteremia (study outcome) was defined by 1 of 5 events occurring after the incident episode of MSSA CRBSI: 1) development of endocarditis (defined according to modified Duke criteria) (18);2) septic thrombophlebitis (defined by persistent MSSA bacteremia for $>72$ hours after the initiation of active therapy associated to the documentation of a thrombus at the site of catheter insertion); 3) septic metastatic infection, including arthritis, spondylitis, infections involving vascular or osteoarticular prostheses (excluding intravascular catheter) or end-organ hematogenous seeding to other locations remote from the primary focus; 4) persistent bacteremia for $>72$ hours; or 5) persistent fever for $>4$ days after extraction of the first blood culture yielding MSSA, provided that alternative causes had been reasonably excluded (19).

\section{Microbiological Methods}

Blood cultures were processed and isolates were identified according to standard techniques at each participating center. The first MSSA isolate from each patient was stored at $-80^{\circ} \mathrm{C}$ until analysis and sent to the central reference laboratory (Department of Microbiology, University Hospital 12 de Octubre, Madrid, Spain). We subsequently subcultured strains twice and performed antimicrobial susceptibility testing by using the E-test method according to manufacturer's recommendations (AB bioMérieux, Solna, Sweden). We tested vancomycin, daptomycin, oxacillin, and linezolid. In addition, the E-test macromethod was performed to screen for heteroresistant vancomycin-intermediate $S$. aureus (hVISA) (20). All the plates were visually read throughout the entire study period by 2 independent observers, who were blinded to each other and to the clinical outcomes. Interobserver discrepancies were resolved by reevaluation and a consensus decision.

\section{Statistical Analysis}

We used the Student unpaired $t$-test to compare normally distributed continuous variables, the Mann-Whitney U test to compare continuous variables with non-normal distribution, and the $\chi^{2}$ and Fisher exact tests to compare proportions as appropriate. Areas under receiving operating characteristic curves for MICs for selected antistaphylococcal agents (on the basis of their discriminative value at the univariate comparisons) were plotted to select the optimal cutoff value in terms of sensitivity (S) and specificity (Sp) to distinguish between patients with or without complicated bacteremia by means of the Youden's J statistic $(\mathrm{J}=\mathrm{S}+\mathrm{Sp}$ $-1)$. We calculated the Pearson correlation coefficient (r) to assess the strength of the linear relationship among E-test MICs for daptomycin and vancomycin. 
To test the effect of high MICs for antistaphylococcal agents and other clinical variables on the occurrence of complicated MSSA CRBSI, we plotted event-free KaplanMeier survival curves and performed comparisons between groups by using the log-rank test. Statistically relevant variables $(p<0.10)$ at the univariate level were entered into multivariate Cox proportional hazard models with a backward stepwise selection, and hazard ratios (HRs) with 95\% CIs were estimated. Some variables considered clinically relevant were also forced into the models regardless of their univariate $p$ values. All statistical tests were 2-tailed, and the threshold of statistical significance was set at $p<0.05$. We used statistical software SPSS version 20.0 (SPSS, Inc., Chicago, IL, USA) to perform calculations of different analysis and generated graphics by using Prism version 6.0 (GraphPad Software Inc., La Jolla, CA, USA). Variables with an unacceptable proportion of missing values $(>30 \%)$ were excluded from the analysis.

\section{Results}

\section{Study Population and Outcome}

Of 108 patients initially recruited, 19 were excluded because they did not fulfill inclusion criteria after revision of the clinical charts ( 13 had received $>72$ hours of treatment with vancomycin and 6 had had a delay of $>3$ days in catheter removal). An additional 6 patients cases were excluded because of inability to recover the MSSA strain for microbiological analysis. Therefore, we included 83 patients in the final analysis; median follow-up was 479 days (range 5 days $-1,014$ days). No variables were excluded because of missing data. None of the included patients had received vancomycin, daptomycin, oxacillin, or linezolid within the 3 months preceding the incidence MSSA CRBSI episode.

We compared the main demographic and clinical characteristics of the 83 patients in the final cohort (Table 1). Catheter removal was performed for all patients within the first 72 hours from sampling of the first blood cultures and, in most (75 [90.6\%]) patients, within the first 48 hours. Antistaphylococcal $\beta$-lactams were used as empiric therapy in 43 patients $(51.8 \%)$, glycopeptides in $32(38.5 \%)$, and daptomycin in $17(20.4 \%)$. Combined therapy was used for $25(31.1 \%)$ patients. Twelve (14.5\%) patients received no empiric therapy or the regimen administered was based on antimicrobial drugs with no in vitro activity against MSSA. For all patients who received daptomycin, the dose used was $\geq 6 \mathrm{mg} / \mathrm{kg} /$ day (range $6-10$ $\mathrm{mg} / \mathrm{kg} /$ day). Venous Doppler ultrasound examination and echocardiogram were performed for $41(49.5 \%)$ and 61 patients $(73.5 \%)$, respectively.

Complicated MSSA CRBSI developed in 26 patients $(31.3 \%)$ at a median of 4 days (interquartile range [IQR] 1-26 days) after sampling of blood cultures. No patients had late complications (i.e., $>30$ days). Septic thrombophlebitis was the most frequent complication (10 patients [12\%]), followed by right-sided endocarditis ( 2 cases [2.4\%]), hematogenous osteoarticular infection (2 cases [2.4\%]), and pulmonary emboli (2 cases [2.4\%]) (online Technical Appendix Table 1, http://wwwnc.cdc.gov/EID/ article/22/6/15-1709-Techapp1.pdf). All-cause and MSSA CRBSI-attributable 30-day mortality rates were $12.0 \%$ and $2.4 \%$, respectively.

\section{Correlation between MICs and Outcome}

All strains were sensitive to all antimicrobial drugs tested. None of the isolates had the hVISA phenotype. The $50 \%$ MIC and 90\% MIC and distribution ranges values were 0.5 $\mu \mathrm{g} / \mathrm{mL}$ and $0.75 \mu \mathrm{g} / \mathrm{mL}$ (range $0.5-0.75 \mu \mathrm{g} / \mathrm{mL}$ ) for cloxacillin, $1.5 \mu \mathrm{g} / \mathrm{mL}$ and $2 \mu \mathrm{g} / \mathrm{mL}$ (range $0.5-2.5 \mu \mathrm{g} / \mathrm{mL}$ ) for vancomycin, $0.38 \mu \mathrm{g} / \mathrm{mL}$ and $0.75 \mu \mathrm{g} / \mathrm{mL}$ (range 0.125 $0.94 \mu \mathrm{g} / \mathrm{mL}$ ) for daptomycin, and $1 \mu \mathrm{g} / \mathrm{mL}$ and $1.5 \mu \mathrm{g} / \mathrm{mL}$ (range $0.25-2 \mu \mathrm{g} / \mathrm{mL}$ ) for linezolid.

We compiled a comparative description of geometric means of MICs determined by E-test for vancomycin, daptomycin, oxacillin, and linezolid among isolates from patients with or without complicated bacteremia (Table 2). We observed that the MICs for vancomycin and daptomycin were higher for isolates from patients in whom complicated bacteremia developed.

We subsequently performed an exploratory area under receiving operating characteristic curve analysis to establish the optimal cutoff point for the daptomycin MIC with the best discriminatory capacity to predict development of complicated bacteremia. An MIC for daptomycin of $0.5 \mu \mathrm{g} / \mathrm{mL}$ was selected (online Technical Appendix Figure 1). This approach also confirmed that 1.5 $\mu \mathrm{g} / \mathrm{mL}$ was the optimal cutoff point for the vancomycin MIC (online Technical Appendix Figure 2). A total of 43 strains $(41.8 \%)$ had a vancomycin $\mathrm{MIC} \geq 1.5, \mu \mathrm{g} / \mathrm{mL}$ and $13(15.7 \%)$ had a daptomycin MIC $>0.5 \mu \mathrm{g} / \mathrm{mL}$. On the basis of these thresholds, $11(84.6 \%)$ of 13 isolates with high MICs for daptomycin also showed high MICs for vancomycin, and we found a moderate positive correlation between these variables $(r=0.21, p=0.05)$ (online Technical Appendix Figure 3). The percentage of MSSA strains with high MICs for vancomycin or daptomycin was higher in the group of patients with complicated bacteremia (Table 2). Strains isolated from all the patients in whom septic thrombophlebitis developed had high MICs for vancomycin or daptomycin.

We compared clinical characteristics of patients according to MICs for vancomycin or daptomycin (Table $3)$. Rates of complicated MSSA CRBSI were significantly higher among patients with episodes caused by strains with MICs for vancomycin $(42 \%$ vs. $20 \%$; $p=0.05)$ or daptomycin $(69.2 \%$ vs. $24.3 \%$; $p=0.004)$ than for those with 
Table 1. Demographics and clinical characteristics of 83 patients with methicillin-sensitive Staphylococcus aureus catheter-related bloodstream infection*

\begin{tabular}{|c|c|}
\hline Variable & Value \\
\hline Age, y & $60 \pm 1.9$ \\
\hline Male sex & $49(59.0)$ \\
\hline \multicolumn{2}{|l|}{ Recruiting center } \\
\hline 1 & $32(38.6)$ \\
\hline 2 & 19 (22.9) \\
\hline 3 & $2(2.4)$ \\
\hline 4 & $12(14.5)$ \\
\hline 5 & $18(21.7)$ \\
\hline \multicolumn{2}{|l|}{ Prognosis of underlying disease } \\
\hline Not fatal & $31(37.3)$ \\
\hline Fatal & $44(53.0)$ \\
\hline Rapidly fatal & $8(9.6)$ \\
\hline Charlson comorbidity index & $3.7 \pm 2.3$ \\
\hline \multicolumn{2}{|l|}{ Previous conditions } \\
\hline Diabetes & $32(38.6)$ \\
\hline Malignancy & $45(54.2)$ \\
\hline Valvular prosthesis & $1(1.2)$ \\
\hline Ostheoarticular prosthesis & $3(3.6)$ \\
\hline Renal failure requiring hemodialysis & $10(12)$ \\
\hline \multicolumn{2}{|l|}{ Type of intravascular catheter } \\
\hline Peripheral venous & $32(38.6)$ \\
\hline Nontunneled (temporary) central venous & $25(30.1)$ \\
\hline Peripherally inserted central & $10(12)$ \\
\hline Permanent central venous & $16(19.3)$ \\
\hline Pitt score at bacteremia onset & $1.6 \pm 1.4$ \\
\hline Severe sepsis or septic shock & $17(20.5)$ \\
\hline \multicolumn{2}{|l|}{ Empiric treatment including $\dagger$} \\
\hline Glycopeptides & $32(38.5)$ \\
\hline Antistaphylococcal $\beta$-lactams $\ddagger$ & $43(51.8)$ \\
\hline Other antistaphylococcal antimicrobial drugs & $4(4.8)$ \\
\hline Daptomycin & $17(20.4)$ \\
\hline None or noneffective antimicrobial drugs & $12(14.5)$ \\
\hline \multicolumn{2}{|l|}{ Antimicrobial regimen } \\
\hline Glycopeptides followed by antistaphylococcal $\beta$-lactam & $25(30.1)$ \\
\hline Only antistaphylococcal $\beta$-lactams & $30(36.1)$ \\
\hline Daptomycin followed by antistaphylococcal $\beta$-lactam & $12(14.5)$ \\
\hline Only daptomycin & $1(1.2)$ \\
\hline Glycopeptides followed by daptomycin plus antistaphylococcal $\beta$-lactam & $7(8.4)$ \\
\hline Daptomycin plus antistaphylococcal $\beta$-lactam & $7(8.4)$ \\
\hline Other & $1(1.2)$ \\
\hline \multicolumn{2}{|l|}{ Timing of catheter removal } \\
\hline Same day or before sampling first blood cultures & $47(56.6)$ \\
\hline 1 day after sampling & $12(14.5)$ \\
\hline 2 days after sampling & $17(20.5)$ \\
\hline 3 days after sampling & $7(8.4)$ \\
\hline Venous Doppler ultrasound examination & $41(49.5)$ \\
\hline Echocardiogram & $61(73.5)$ \\
\hline Complicated MSSA CRBSI & $26(31.3)$ \\
\hline Persistent fever $>4 \mathrm{~d} \S$ & $11(13.3)$ \\
\hline Persistent bacteremia $>72 \mathrm{~h}$ ा & $6(7.2)$ \\
\hline Septic thrombophlebitis & $10(12)$ \\
\hline Endocarditis & $2(2.4)$ \\
\hline Hematogenous osteoarticular infection & $2(2.4)$ \\
\hline Pulmonary emboli & $2(2.4)$ \\
\hline All-cause deaths at 30 days & $10(12)$ \\
\hline MSSA CRBSI-attributable deaths at 30 days & $2(2.4)$ \\
\hline 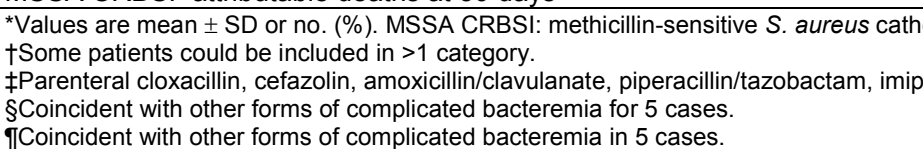 & \\
\hline
\end{tabular}

episodes caused by strains with lower MICs. Strains with high MICs for vancomycin were not associated with higher rates of severe sepsis or septic shock at bacteremia onset, or with increased all-cause or attributable mortality rates. Conversely, we found that high MICs for daptomycin were more common among hemodynamically unstable episodes 
Table 2. Antimicrobial susceptibility testing (E-test) of isolates from patients with methicillin-sensitive Staphylococcus aureus catheterrelated bloodstream infection with or without complicated bacteremia*

\begin{tabular}{|c|c|c|}
\hline \multirow[b]{2}{*}{ Variable } & \multicolumn{2}{|c|}{ Complicated bacteremia } \\
\hline & No, $n=57$ & Yes, $n=26$ \\
\hline \multicolumn{3}{|l|}{$\mathrm{MIC}, \mu \mathrm{g} / \mathrm{mL}$} \\
\hline Vancomycin & $1.2 \pm 0.4 \dagger$ & $1.5 \pm 0.48 \dagger$ \\
\hline Oxacillin & $0.49 \pm 0.27$ & $0.51 \pm 0.2$ \\
\hline Daptomycin & $0.4 \pm 0.16 \dagger$ & $0.5 \pm 0.2 \dagger$ \\
\hline Linezolid & $1.08 \pm 0.13$ & $1.05 \pm 0.08$ \\
\hline \multicolumn{3}{|l|}{ Frequency } \\
\hline Vancomycin $\mathrm{MIC} \geq 1.5 \mu \mathrm{g} / \mathrm{mL}$ & $25(43.9) \dagger$ & $18(69.2) \dagger$ \\
\hline Daptomycin MIC $>0.5 \mu \mathrm{g} / \mathrm{mL}$ & $4(7.0) \dagger$ & $9(34.6) \dagger$ \\
\hline Vancomycin MIC $\geq 1.5 \mu \mathrm{g} / \mathrm{mL}$ or daptomycin $\mathrm{MIC}>0.5 \mu \mathrm{g} / \mathrm{mL}$ & $26(45.6) \dagger$ & $19(73.1) \dagger$ \\
\hline Vancomycin MIC $\geq 1.5 \mu \mathrm{g} / \mathrm{mL}$ and daptomycin $\mathrm{MIC}>0.5 \mu \mathrm{g} / \mathrm{mL}$ & $3(5.3) \dagger$ & $8(30.8) \dagger$ \\
\hline
\end{tabular}

(i.e., severe sepsis or septic shock) than among remaining episodes $(46.2 \%$ vs. $15.7 \%, \mathrm{p}=0.02)$. However, no effect on mortality rates was observed.

\section{Risk Factors for Development of Complicated Bacteremia}

We compared clinical characteristics of patients with or without complicated bacteremia (Table 4). Most (65.4\%) patients with complicated cases were given a diagnosis at 1 recruiting center. Such an imbalance might be partially explained by the fact that such a center was the study promoter and the largest center at which the greatest diagnostic efforts were made. The probability of remaining free of complicated bacteremia at day 30 was significantly lower for patients with MSSA CRBSI caused by isolates with daptomycin MICs $>0.5 \mu \mathrm{g} / \mathrm{mL}$ (33. $3 \%$ vs. $75 \%$; log-rank test $\mathrm{p}=0.002)$ and isolates with vancomycin MICs $\geq 1.5$ $\mu \mathrm{g} / \mathrm{mL}(59.2 \%$ vs. $79.6 \%$; log-rank test $\mathrm{p}=0.02)($ Figure $)$.

Because of low number of patients with complicated bacteremia (26 episodes), we could not assess the potential effect of MICs by performing a single logistic regression model adjusted for all the covariates found to be significant by univariate analysis. We alternatively attempted an exploratory approach based on different models. We used Cox regression models that incorporated a maximum of 3 variables at a time, always including the recruiting center as a potential confounder (Table 5; online Technical Appendix Table 2). The presence of high MICs for vancomycin (minimum adjusted HR 2.4, 95\% CI 1.2-5.5) and daptomycin (minimum adjusted HR 2.4, 95\% CI 1.1-5.9) were kept as independent risk factors for development of complicated MSSA CRBSI in all models.

We performed a sensitivity analysis by restricting the study outcome to the occurrence of septic thrombophlebitis. These associations remained essentially unchanged, and we observed even higher HRs (minimum adjusted HR for vancomycin MIC 6.0 [95\% CI 0.7-52.2]; minimum adjusted HR for daptomycin MIC 5.5 [95\% CI 1.3-22.5]) (online Technical Appendix Tables 2, 3).

\section{Discussion}

The aim of our study was to investigate the potential role of high MICs for vancomycin and other antistaphylococcal agents in predicting the risk for complications in a selected cohort of patients with MSSA CRBSI. We investigated this role after controlling for other well-defined prognostic factors, such as timing of catheter removal or appropriateness of empiric therapy.

We found a correlation between high MICs for vancomycin $(\geq 1.5 \mu \mathrm{g} / \mathrm{mL})$ or daptomycin $(>0.5 \mu \mathrm{g} / \mathrm{mL})$ and an increased incidence of complicated bacteremia, particularly in terms of local endovascular complications represented by septic thrombophlebitis. Venous Doppler ultrasound examination was performed for only $50 \%$ of patients. Notwithstanding this potential drawback, we included in the definition of the study outcome the presence of persistent bacteremia in the absence of proven hematogenous spread to distant locations. This criterion might act as an appropriate clinical surrogate for identifying potential cases of septic thrombophlebitis that could have eventually remained undiagnosed.

Results confirm those obtained in our previous singlecenter retrospective study (13), in which we demonstrated that MSSA strains with vancomycin MICs $\geq 1.5 \mu \mathrm{g} / \mathrm{mL}$ were associated with a $>2$-fold increase in risk for development of complicated bacteremia and, specifically, a $>6$-fold increase in the risk for development of septic thrombophlebitis. In both studies, and in contrast to other authors who evaluated MSSA bacteremia from different sources (7), including patients with endocarditis (8), we did not find any apparent effect of increased MICs on sepsis severity or risk for death. The relatively low 30-day mortality rate for our selected cohort could partially account for this discrepancy. However, López-Cortés et al. (12) did not demonstrate any apparent effect of MICs for vancomycin on mortality rates in a recent study of a nonselected cohort of patients with MSSA bacteremia. These authors also reported an increased incidence of septic thrombophlebitis among patients with MSSA bacteremia caused by strains 
Table 3. Demographic and clinical characteristics of patients with methicillin-sensitive Staphylococcus aureus catheter-related bloodstream infection by MICs for vancomycin and daptomycin measured by E-test*

\begin{tabular}{|c|c|c|c|c|c|c|}
\hline \multirow[b]{2}{*}{ Variable } & \multicolumn{3}{|c|}{ Vancomycin MIC, $\mu \mathrm{g} / \mathrm{mL}$} & \multicolumn{3}{|c|}{ Daptomycin MIC, $\mu \mathrm{g} / \mathrm{mL}$} \\
\hline & $<1.5, n=40$ & $\geq 1.5, n=43$ & $p$ value & $\leq 0.5, \mathrm{n}=70$ & $>0.5, \mathrm{n}=13$ & $p$ value \\
\hline Age, y & $61.9 \pm 16.0$ & $59.9 \pm 19.0$ & 0.42 & $60.2 \pm 17.3$ & $63.8 \pm 21.0$ & 0.5 \\
\hline Male sex & $23(57.5)$ & $26(60.5)$ & 0.42 & $41(58.6)$ & $8(61.5)$ & 0.4 \\
\hline \multicolumn{7}{|l|}{ Prognosis of underlying disease } \\
\hline Not fatal & $18(45.0)$ & $13(30.2)$ & 0.3 & $24(34.3)$ & $7(53.8)$ & 0.3 \\
\hline Fatal & $17(42.5)$ & $27(62.8)$ & 0.1 & $39(55.7)$ & $5(38.5)$ & 0.4 \\
\hline Rapidly fatal & $5(12.5)$ & $3(6.9)$ & 0.6 & $7(10.0)$ & $1(7.7)$ & 0.8 \\
\hline Charlson comorbidity index & $3.5 \pm 2.6$ & $4.0 \pm 2.1$ & 0.31 & $3.7 \pm 2.3$ & $3.5 \pm 2.1$ & 0.9 \\
\hline \multicolumn{7}{|l|}{ Previous conditions } \\
\hline Diabetes & $20(50.0)$ & $12(27.9)$ & 0.06 & $28(40)$ & $4(30.8)$ & 0.6 \\
\hline Malignancy & $17(42.5)$ & $28(65.1)$ & 0.12 & $37(52.9)$ & $8(61.5)$ & 0.5 \\
\hline Valvular prosthesis & $0(0.0)$ & $1(2.3)$ & 0.9 & $1(1.4)$ & $0(0.0)$ & 0.9 \\
\hline Ostheoarticular prosthesis & $1(2.5)$ & $2(4.7)$ & 0.79 & $2(2.9)$ & $1(7.7)$ & 0.6 \\
\hline Renal failure requiring hemodialysis & $7(17.5)$ & $3(6.9)$ & 0.2 & $9(12.9)$ & $1(7.7)$ & 0.5 \\
\hline \multicolumn{7}{|l|}{ Type of intravascular catheter } \\
\hline Peripheral venous & $17(42.5)$ & $15(34.9)$ & 0.71 & $28(40)$ & $4(30.8)$ & 0.7 \\
\hline Nontunneled (temporary) central venous & $13(32.5)$ & $12(27.9)$ & 0.82 & $19(27.1)$ & $6(46.2)$ & 0.3 \\
\hline Peripherally inserted central & $5(12.5)$ & $5(11.6)$ & 0.76 & $10(14.3)$ & $0(0.0)$ & 0.3 \\
\hline Permanent central venous & $5(12.5)$ & $11(25.6)$ & 0.2 & $13(18.6)$ & $3(23.1)$ & 0.6 \\
\hline Pitt score at bacteremia onset & $1.5 \pm 1.7$ & $1.1 \pm 1.4$ & 0.9 & $1.3 \pm 1.7$ & $1.2 \pm 1.0$ & 0.9 \\
\hline Severe sepsis or septic shock & $11(27.5)$ & $6(13.9)$ & 0.2 & $11(15.7)$ & $6(46.2)$ & 0.02 \\
\hline \multicolumn{7}{|l|}{ Empiric treatment including $†$} \\
\hline Glycopeptides & $16(40.0)$ & $16(37.2)$ & 0.7 & 27 (38.5) & $5(38.4)$ & 0.7 \\
\hline Antistaphylococcal $\beta$-lactams $\ddagger$ & $21(52.5)$ & $22(51.1)$ & 0.9 & $37(52.8)$ & $6(46.1)$ & 0.7 \\
\hline Other antistaphylococcal antimicrobial drugs & $1(2.5)$ & $3(6.9)$ & 0.7 & $4(5.7)$ & $0(0.0)$ & 0.8 \\
\hline Daptomycin & $7(17.5)$ & $10(23.2)$ & 0.8 & $13(18.5)$ & $4(30.7)$ & 0.5 \\
\hline None or noneffective antimicrobial drugs & $7(17.5)$ & $5(11.6)$ & 0.8 & $10(14.3)$ & $2(15.4)$ & 0.8 \\
\hline \multicolumn{7}{|l|}{ Antimicrobial regimen } \\
\hline $\begin{array}{l}\text { Glycopeptides followed by antistaphylococcal } \\
\beta \text {-lactam }\end{array}$ & $11(27.5)$ & $14(32.6)$ & 0.7 & $21(30.0)$ & $4(30.8)$ & 0.9 \\
\hline Only antistaphylococcal $\beta$-lactams & $14(35)$ & $16(37.2)$ & 0.8 & $26(37.1)$ & $4(30.8)$ & 0.8 \\
\hline Daptomycin followed by antistaphylococcal $\beta$-lactam & $3(7.5)$ & $9(20.9)$ & 0.16 & $8(11.4)$ & $4(30.8)$ & 0.2 \\
\hline Only daptomycin & $1(2.5)$ & $0(0.0)$ & 0.9 & $1(1.5)$ & $0(0.0)$ & 0.9 \\
\hline $\begin{array}{l}\text { Glycopeptides followed by daptomycin plus } \\
\text { antistaphylococcal } \beta \text {-lactam }\end{array}$ & $6(15.0)$ & $1(2.3)$ & 0.09 & $6(8.6)$ & $1(7.7)$ & 0.9 \\
\hline Daptomycin plus antistaphylococcal $\beta$-lactam & $4(10.0)$ & $3(6.9)$ & 0.8 & $7(10)$ & $0(0.0)$ & 0.5 \\
\hline Other & $1(1.25)$ & $0(0.0)$ & 0.6 & $1(2.5)$ & $0(0.0)$ & 0.7 \\
\hline \multicolumn{7}{|l|}{ Timing of catheter removal } \\
\hline Same day or before sampling first blood cultures & $28(70.0)$ & $19(44.2)$ & 0.03 & $41(58.6)$ & $6(46.2)$ & 0.7 \\
\hline 1 day after sampling & $6(15.0)$ & $6(13.9)^{\prime}$ & 0.9 & $10(14.3)$ & $2(15.4)$ & 0.9 \\
\hline 2 days after sampling & $5(12.5)$ & $12(27.9)$ & 0.1 & $12(17.1)$ & $5(38.5)$ & 0.2 \\
\hline 3 days after sampling & $1(2.5)$ & $6(13.9)$ & 0.1 & $7(10.0)$ & $0(0.0)$ & 0.6 \\
\hline Complicated MSSA CRBSI & $8(20.0)$ & $18(41.9)$ & 0.05 & $17(24.3)$ & $9(69.2)$ & 0.004 \\
\hline Persistent fever for $\geq 4 \mathrm{~d}$ & $3(7.5)$ & $8(18.6)$ & 0.2 & $9(12.9)$ & $2(15.4)$ & 0.8 \\
\hline Persistent bacteremia for $\geq 72 \mathrm{~h}$ & $2(5.0)$ & $4(9.3)$ & 0.7 & $5(7.1)$ & $1(7.7)$ & 0.9 \\
\hline Septic thrombophlebitis & $1(2.5)$ & $9(20.9)$ & 0.02 & $4(5.7)$ & $6(46.2)$ & $<0.0001$ \\
\hline Endocarditis & $1(2.5)$ & $1(2.3)$ & 0.9 & $2(2.9)$ & $0(0.0)$ & 0.9 \\
\hline Hematogenous osteoarticular infection & $1(2.5)$ & $1(2.3)$ & 0.9 & $1(1.4)$ & $1(7.7)$ & 0.5 \\
\hline Pulmonary emboli & $2(4.7)$ & $0(0.0)$ & 0.3 & $2(2.9)$ & $0(0.0)$ & 0.6 \\
\hline All-cause deaths at 30 days & $6(15.0)$ & $4(9.3)$ & 0.5 & $9(12.9)$ & $1(7.7)$ & 0.5 \\
\hline MSSA CRBSI-attributable deaths at 30 days & $1(2.5)$ & $1(2.3)$ & 0.4 & $2(2.9)$ & $0(0.0)$ & 0.4 \\
\hline
\end{tabular}

with high MICs for vancomycin. This combined evidence suggests that the theoretically increased pathogenicity of these strains might exert a local vascular effect but does necessarily imply a higher risk for severe sepsis or death.

The role of MICs for daptomycin as a prognostic marker in episodes of MSSA bacteremia has not been extensively investigated. In a study by Cervera et al., which included only patients with endocarditis caused by MSSA,
MICs for daptomycin were not related to increased mortality rates (8). We have established a cutoff value for the MIC for daptomycin $(>0.5 \mu \mathrm{g} / \mathrm{mL})$ that also identified patients with an increased risk for development of complicated MSSA CRBSI, particularly septic thrombophlebitis. In accordance with lack of a correlation for the MIC for vancomycin, mortality rates did not differ according to MICs for daptomycin. Only 11 isolates (13.2\% of the cohort) had 
Table 4. Comparative analysis of patients with methicillin-sensitive Staphylococcus aureus catheter-related bloodstream infection with or without complicated bacteremia*

\begin{tabular}{|c|c|c|c|}
\hline \multirow[b]{2}{*}{ Variable } & \multicolumn{2}{|c|}{ Complicated bacteremia } & \multirow[b]{2}{*}{$p$ value } \\
\hline & No, $n=5$ & Yes, $n=26$ & \\
\hline Age, y & $61.4 \pm 16.0$ & $59.3 \pm 21.0$ & 0.1 \\
\hline Male sex & $32(56.1)$ & $17(65.4)$ & 0.5 \\
\hline \multicolumn{4}{|l|}{ Recruiting center } \\
\hline 1 & $15(26.3)$ & $17(65.4)$ & 0.01 \\
\hline 2 & $14(24.6)$ & $5(19.2)$ & 0.8 \\
\hline 3 & $2(3.5)$ & $0(0.0)$ & 1.0 \\
\hline 4 & $11(19.3)$ & $1(3.8)$ & 0.2 \\
\hline 5 & $15(26.3)$ & $3(11.5)$ & 0.2 \\
\hline \multicolumn{4}{|l|}{ Prognosis of underlying disease } \\
\hline Not fatal & $24(42.1)$ & $7(26.9)$ & 0.2 \\
\hline Fatal & $29(50.9)$ & $15(57.7)$ & 0.6 \\
\hline Rapidly fatal & $4(7.0)$ & $4(15.4)$ & 0.3 \\
\hline Charlson comorbidity index & $3.6 \pm 2.4$ & $1.9 \pm 1.9$ & 0.3 \\
\hline \multicolumn{4}{|l|}{ Previous conditions } \\
\hline Diabetes & $20(35.1)$ & $12(46.2)$ & 0.3 \\
\hline Malignancy & $29(50.9)$ & $16(61.5)$ & 0.6 \\
\hline Valvular prosthesis & $1(1.8)$ & $0(0.0)$ & 1.0 \\
\hline Ostheoarticular prosthesis & $1(1.8)$ & $2(7.7)$ & 0.2 \\
\hline Renal failure requiring hemodialysis & $7(12.3)$ & $3(11.5)$ & 1.0 \\
\hline \multicolumn{4}{|l|}{ Type of intravascular catheter } \\
\hline Peripheral venous & $23(40.4)$ & $9(34.6)$ & 0.8 \\
\hline Nontunneled (temporary) central venous & $17(29.8)$ & $8(30.8)$ & 1.0 \\
\hline Peripherally inserted central & $7(12.3)$ & $3(11.5)$ & 1.0 \\
\hline Permanent central venous & $10(17.5)$ & $6(23.1)$ & 0.6 \\
\hline Pitt score at bacteremia onset & $1.1 \pm 1.4$ & $1.7 \pm 1.9$ & 0.1 \\
\hline Severe sepsis or septic shock & $11(19.3)$ & $6(23.1)$ & 0.3 \\
\hline \multicolumn{4}{|l|}{ Empiric treatment includingt } \\
\hline Glycopeptides & $24(42)$ & $8(30.7)$ & 0.4 \\
\hline Antistaphylococcal $\beta$-lactams $\ddagger$ & $31(54.3)$ & $12(46.1)$ & 0.6 \\
\hline Other antistaphylococcal antimicrobial drugs & $1(1.7)$ & $3(11.5)$ & 0.2 \\
\hline Daptomycin & $8(14)$ & $9(34.6)$ & 0.06 \\
\hline Daptomycin monotherapy & $5(8.8)$ & $8(30.8)$ & 0.02 \\
\hline None or noneffective antimicrobial drugs & $10(17.5)$ & $2(7.7)$ & 0.4 \\
\hline \multicolumn{4}{|l|}{ Antimicrobial regimen } \\
\hline Glycopeptides followed by antistaphylococcal $\beta$-lactam & $19(33.3)$ & $6(23.1)$ & 0.4 \\
\hline Only antistaphylococcal $\beta$-lactams & $23(40.4)$ & $7(26.9)$ & 0.3 \\
\hline Daptomycin followed by antistaphylococcal $\beta$-lactam & $4(7.0)$ & $8(30.8)$ & 0.01 \\
\hline Only daptomycin & $1(1.8)$ & $0(0.0)$ & 1.0 \\
\hline Glycopeptides followed by daptomycin plus antistaphylococcal $\beta$-lactam & $6(10.5)$ & $1(3.8)$ & 0.4 \\
\hline Daptomycin plus antistaphylococcal $\beta$-lactam & $3(5.3)$ & $4(15.4)$ & 0.2 \\
\hline Other & $1(1.8)$ & $0(0.0)$ & 1.0 \\
\hline \multicolumn{4}{|l|}{ Timing of catheter removal } \\
\hline Same day or before of sampling first blood cultures & $36(63.2)$ & $11(42.3)$ & 0.1 \\
\hline 1 day after sampling & $6(10.5)$ & $6(23.1)$ & 0.1 \\
\hline 2 days after sampling & $10(17.5)$ & $7(26.9)$ & 0.4 \\
\hline 3 days after sampling & $5(8.8)$ & $2(7.7)$ & 1.0 \\
\hline Venous Doppler ultrasound examination & $21(36.8)$ & $20(76.9)$ & 0.001 \\
\hline Echocardiogram & $36(63.2)$ & $25(96.2)$ & 0.01 \\
\hline All-cause deaths at 30 days & $7(12.3)$ & $3(11.5)$ & 1.0 \\
\hline MSSA CRBSI-attributable deaths at 30 days & $0(0.0)$ & $2(7.7)$ & 0.09 \\
\hline
\end{tabular}

simultaneously high MICs for vancomycin and daptomycin, and multivariate analysis demonstrated that both variables had an independent effect on the risk for development of complicated MSSA CRBSI.

It has been reported that MRSA isolates, mostly hVISA isolates, with higher MICs for vancomycin also have increased MICs for daptomycin (21-24). However, other studies have failed to confirm such an association (25-27).
Pillai et al. found that some MSSA strains obtained from a patient with persistent bacteremia treated with vancomycin showed increasing MICs for daptomycin (28). In vitro studies suggested that patients infected with MSSA strains that have high MICs for vancomycin could have poor responses not only to vancomycin (29) but also to other antistaphylococcal agents, such as cloxacillin or daptomycin (30). In a recent in vitro study, daptomycin 

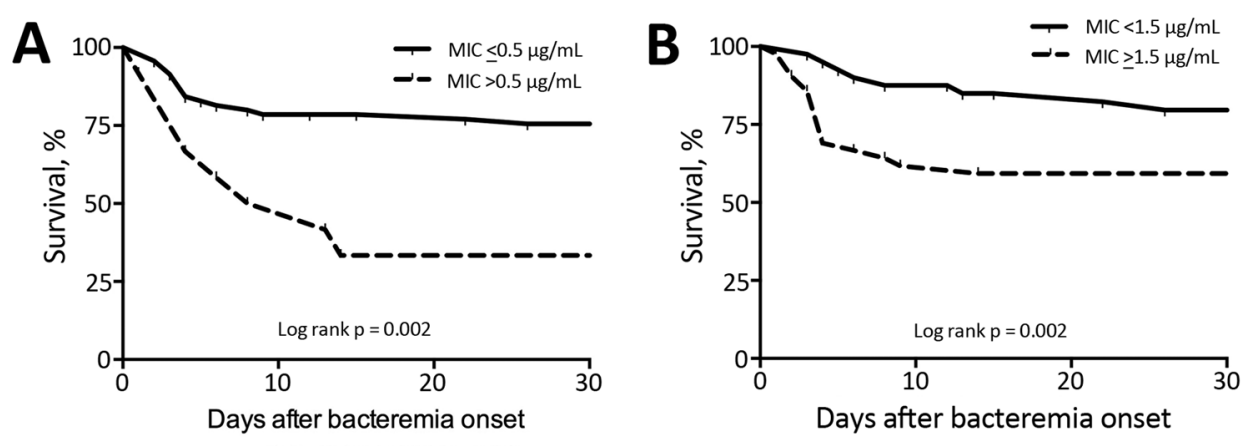

Figure. Event-free Kaplan-Meier survival curves for complicated bacteremia according to E-test MICs for daptomycin (A) and vancomycin (B) for complicated catheter-related bloodstream infections with a methicillinsensitive Staphylococcus aureus isolate.

resistance was associated with increased thickness of bacterial cell wall peptidoglycan and a slightly delayed transition to the postexponential growth phase because of alterations in bacterial metabolism (31). These findings suggest a deleterious fitness cost in terms of invasiveness (31) but also a blockage in the adhesive phase of $S$. aureus that might be linked to an increased trend in local complications. High MICs for vancomycin in isolates with the hVISA phenotype have been shown to be associated with increased persistence, rather than increased mortality rates and sepsis in patients (32). Although we did not detect any hVISA isolates in our study, we postulate that a high MIC for vancomycin could be an essential step in acquisition of this phenotype, and that this step might be the common underlying pathogenic mechanism.

Apart from the limited number of events (which prevented including all explanatory variables into a single multivariate model), some limitations of the study also deserve specific consideration. Despite its multicenter nature, 1 specific participating center provided most of the results because this center recruited most of the patients and most patients who had complicated bacteremia. However, all multivariate models were adjusted by the recruiting center to minimize such potential bias. Antimicrobial drug susceptibility testing was not performed in parallel with the reference broth microdilution method, which could have enabled us to confirm the poor correlation previously reported between this technique and the E-test (11).

Conversely, interobserver reproducibility of the Etest for assessing vancomycin MICs has been recently questioned (33). In our study, MIC testing was performed over a continuous period, and E-test results were read by 2 independent observers who were blinded to patient outcome, an approach that theoretically would reduce intraassay variability and facilitate interpretation of results. Finally, real-life applicability of our findings and potential prognostic value of performing E-tests for daptomycin for all MSSA isolates from patients with CRBSI remain to be established with support of cost-effectiveness analysis. Although we did not specifically address some variables that had been postulated to be related with higher MICs for vancomycin or daptomycin, such as inoculum size (34) or use of suboptimal dosing of daptomycin for cases of persistent bacteremia (35), we set a priori inclusion criteria to homogenize the clinical management of patients analyzed (including uniform dosing of daptomycin and vancomycin).

In summary, our data suggest that patients with MSSA CRBSI caused by strains with MICs $\geq 1.5 \mu \mathrm{g} / \mathrm{mL}$ for vancomycin or $>0.5 \mu \mathrm{g} / \mathrm{mL}$ for daptomycin determined by E-test are more prone to show development of complications, particularly septic thrombophlebitis. However, these patients do not appear to have an increased risk for severe sepsis or death. On the basis of these preliminary findings, patients with CRBSI cause by MSSA isolates with high MICs for vancomycin or daptomycin should be carefully evaluated to exclude local complications and eventually individualize duration of therapy. These prospects would merit confirmation in future intervention studies.

\section{Acknowledgments}

We thank Mercedes Fernández de Mera for providing assistance with microbiological techniques.

Table 5. Univariate and multivariate analyses of risk factors for development of complicated methicillin-sensitive Staphylococcus aureus catheter-related bloodstream infection*

\begin{tabular}{lcc}
\hline Variable & Univariate, $\mathrm{HR}(95 \% \mathrm{Cl})$ & Multivariate, $\mathrm{HR}(95 \% \mathrm{Cl}) \dagger$ \\
\hline Recruiting center 1 & $3.6(1.6-8.1)$ & - \\
Any daptomycin-containing empiric therapy & $2.5(1.1-5.7)$ & - \\
Daptomycin monotherapy as empiric therapy & $3.1(1.3-7.1)$ & - \\
Vancomycin MIC $\geq 1.5 \mu \mathrm{g} / \mathrm{mL}$ & $2.6(1.1-5.9)$ & $2.4(1.2-5.5)$ \\
Daptomycin MIC $>0.5 \mu \mathrm{g} / \mathrm{mL}$ & $3.4(1.6-8.2)$ & $2.4(1.1-5.9)$ \\
\hline
\end{tabular}

Daptomycin MIC $>0.5 \mu \mathrm{g} / \mathrm{mL}$

${ }^{*} \mathrm{HR}$, hazard ratio; -, corresponding variables were not retained in the final model.

†Various models, including a maximum of 3 variables, were explored that combined all statistically significant variables identified at the univariate level.

Only variables that were constantly retained in these models are shown with minimum HR values obtained. 
This study was supported by the Spanish Ministry of Economy and Competitiveness, Instituto de Salud Carlos III, Agency for Health Technology Assessment and Research (PS11/01229); Plan Nacional de Investigación Científica, Desarrollo e Innovación Tecnológica, 2008-2011, Spanish Network for Research in Infectious Diseases (REIPI RD12/0015); and a European Development Regional Fund project (A Way to Achieve Europe). M.F.-R. is supported by clinical research contract Juan Rodés (JR14/00036) from the Spanish Ministry of Economy and Competitiveness, Instituto de Salud Carlos III.

Dr. San-Juan is clinical microbiologist at the Unit of Infectious Diseases of the University Hospital 12 de Octubre, Madrid, Spain. His primary research interests are clinical and microbiological aspects of Staphylococcus aureus infections, as well as infectious complications in solid-organ transplant recipients.

\section{References}

1. Wyllie DH, Crook DW, Peto TE. Mortality after Staphylococcus aureus bacteraemia in two hospitals in Oxfordshire, 1997-2003: cohort study. BMJ. 2006;333:281. http://dx.doi.org/10.1136/ bmj.38834.421713.2F

2. Lodise TP, Graves J, Evans A, Graffunder E, Helmecke M, Lomaestro BM, et al. Relationship between vancomycin MIC and failure among patients with methicillin-resistant Staphylococcus aureus bacteremia treated with vancomycin. Antimicrob Agents Chemother. 2008;52:3315-20. http://dx.doi.org/ 10.1128/AAC.00113-08

3. Moise PA, Sakoulas G, Forrest A, Schentag JJ. Vancomycin in vitro bactericidal activity and its relationship to efficacy in clearance of methicillin-resistant Staphylococcus aureus bacteremia. Antimicrob Agents Chemother. 2007;51:2582-6. http://dx.doi.org/10.1128/ AAC.00939-06

4. Sakoulas G, Moise-Broder PA, Schentag J, Forrest A, Moellering RC Jr, Eliopoulos GM. Relationship of MIC and bactericidal activity to efficacy of vancomycin for treatment of methicillin-resistant Staphylococcus aureus bacteremia. J Clin Microbiol. 2004;42:2398-402. http://dx.doi.org/10.1128/ JCM.42.6.2398-2402.2004

5. Soriano A, Marco F, Martinez JA, Pisos E, Almela M, Dimova VP, et al. Influence of vancomycin minimum inhibitory concentration on the treatment of methicillin-resistant Staphylococcus aureus bacteremia. Clin Infect Dis. 2008;46:193200. http://dx.doi.org/10.1086/524667

6. van Hal SJ, Lodise TP, Paterson DL. The clinical significance of vancomycin minimum inhibitory concentration in Staphylococcus aureus infections: a systematic review and meta-analysis. Clin Infect Dis. 2012;54:755-71. http://dx.doi.org/10.1093/cid/cir935

7. Holmes NE, Turnidge JD, Munckhof WJ, Robinson JO, Korman TM, O'Sullivan MV, et al. Antibiotic choice may not explain poorer outcomes in patients with Staphylococcus aureus bacteremia and high vancomycin minimum inhibitory concentrations. J Infect Dis. 2011;204:340-7. http://dx.doi.org/ 10.1093/infdis/jir270

8. Cervera C, Castaneda X, de la Maria CG, del Rio A, Moreno A, Soy D, et al. Effect of vancomycin minimal inhibitory concentration on the outcome of methicillin-susceptible Staphylococcus aureus endocarditis. Clin Infect Dis. 2014; 58:1668-75.

9. Gasch O, Camoez M, Dominguez MA, Padilla B, Pintado V, Almirante B, et al. Predictive factors for mortality in patients with methicillin-resistant Staphylococcus aureus bloodstream infection: impact on outcome of host, microorganism and therapy. Clin Microbiol Infect. 2013;19:1049-57. http://dx.doi.org/ 10.1111/1469-0691.12108

10. Lalueza A, Chaves F, San Juan R, Daskalaki M, Otero JR, Aguado JM. Is high vancomycin minimum inhibitory concentration a good marker to predict the outcome of methicillin-resistant Staphylococcus aureus bacteremia? J Infect Dis. 2010;201:311-2, author reply 2-3. http://dx.doi.org/10.1086/649572

11. Rojas L, Bunsow E, Munoz P, Cercenado E, Rodriguez-Creixems M, Bouza E. Vancomycin MICs do not predict the outcome of methicillin-resistant Staphylococcus aureus bloodstream infections in correctly treated patients. J Antimicrob Chemother. 2012; 67:1760-8. http://dx.doi.org/10.1093/jac/dks128

12. López-Cortés LE, Velasco C, Retamar P, Del Toro MD, Galvez-Acebal J, de Cueto M, et al. Is reduced vancomycin susceptibility a factor associated with poor prognosis in MSSA bacteraemia? J Antimicrob Chemother. 2015;70:2652-60. http://dx.doi.org/10.1093/jac/dkv133

13 Aguado JM, San-Juan R, Lalueza A, Sanz F, Rodriguez-Otero J, Gomez-Gonzalez C, et al. High vancomycin MIC and complicated methicillin-susceptible Staphylococcus aureus bacteremia. Emerg Infect Dis. 2011;17:1099-102. http://dx.doi.org/ 10.3201/eid/1706.101037

14. Mermel LA, Allon M, Bouza E, Craven DE, Flynn P, O'Grady NP, et al. Clinical practice guidelines for the diagnosis and management of intravascular catheter-related infection: 2009 Update by the Infectious Diseases Society of America. Clin Infect Dis. 2009;49:1-45. http://dx.doi.org/10.1086/599376

15. Charlson ME, Sax FL, MacKenzie CR, Braham RL, Fields SD, Douglas RG Jr. Morbidity during hospitalization: can we predict it? J Chronic Dis. 1987;40:705-12. http://dx.doi.org/10.1016/ 0021-9681(87)90107-X

16. McCabe WR, Jackson GG. Treatment of chronic pyelonephritis. III. Comparison of several drugs combined and one member of the combination, colistin. Am J Med Sci. 1960;240:754-63. http://dx.doi.org/10.1097/00000441-196012000-00010

17. Chow JW, Yu VL. Combination antibiotic therapy versus monotherapy for gram-negative bacteraemia: a commentary. Int J Antimicrob Agents. 1999;11:7-12. http://dx.doi.org/10.1016/ S0924-8579(98)00060-0

18. Durack DT, Lukes AS, Bright DK. New criteria for diagnosis of infective endocarditis: utilization of specific echocardiographic findings. Duke Endocarditis Service. Am J Med. 1994;96:200-9. http://dx.doi.org/10.1016/0002-9343(94)90143-0

19. Fowler VG Jr, Olsen MK, Corey GR, Woods CW, Cabell CH, Reller LB, et al. Clinical identifiers of complicated Staphylococcus aureus bacteremia. Arch Intern Med. 2003;163:2066-72. http://dx.doi.org/10.1001/archinte.163.17.2066

20. Walsh TR, Bolmstrom A, Qwarnstrom A, Ho P, Wootton M, Howe RA, et al. Evaluation of current methods for detection of staphylococci with reduced susceptibility to glycopeptides. J Clin Microbiol. 2001;39:2439-44. http://dx.doi.org/10.1128/ JCM.39.7.2439-2444.2001

21. Sakoulas G, Alder J, Thauvin-Eliopoulos C, Moellering RC Jr, Eliopoulos GM. Induction of daptomycin heterogeneous susceptibility in Staphylococcus aureus by exposure to vancomycin. Antimicrob Agents Chemother. 2006;50:1581-5. http://dx.doi.org/10.1128/AAC.50.4.1581-1585.2006

22. Cui L, Tominaga E, Neoh HM, Hiramatsu K. Correlation between reduced daptomycin susceptibility and vancomycin resistance in vancomycin-intermediate Staphylococcus aureus. Antimicrob Agents Chemother. 2006;50:1079-82. http://dx.doi.org/10.1128/ AAC.50.3.1079-1082.2006

23. Chen YH, Liu CY, Ko WC, Liao CH, Lu PL, Huang CH, et al. Trends in the susceptibility of methicillin-resistant Staphylococcus aureus to nine antimicrobial agents, including ceftobiprole, 
nemonoxacin, and tyrothricin: results from the tigecycline in vitro surveillance in Taiwan (TIST) study, 2006-2010.

Eur J Clin Microbiol Infect Dis. 2014;33:233-9. http://dx.doi.org/ 10.1007/s10096-013-1949-y

24. Maor Y, Belausov N, Ben-David D, Smollan G, Keller N, Rahav G. hVISA and MRSA endocarditis: an 8-year experience in a tertiary care centre. Clin Microbiol Infect. 2014;20:0730-6. http://dx.doi.org/10.1111/1469-0691.12498

25. Malli E, Spiliopoulou I, Kolonitsiou F, Klapsa D, Giannitsioti E, Pantelidi K, et al. In vitro activity of daptomycin against Gram-positive cocci: the first multicentre study in Greece. Int J Antimicrob Agents. 2008;32:525-8. http://dx.doi.org/10.1016/ j.ijantimicag.2008.05.020

26. Sader HS, Becker HK, Moet GJ, Jones RN. Antimicrobial activity of daptomycin tested against Staphylococcus aureus with vancomycin MIC of 2 microg $/ \mathrm{mL}$ isolated in the United States and European hospitals (2006-2008). Diagn Microbiol Infect Dis. 2010;66:32931. http://dx.doi.org/10.1016/j.diagmicrobio.2009.09.017

27. Rybak MJ, Hershberger E, Moldovan T, Grucz RG. In vitro activities of daptomycin, vancomycin, linezolid, and quinupristin-dalfopristin against staphylococci and enterococci, including vancomycin-intermediate and -resistant strains. Antimicrob Agents Chemother. 2000;44:1062-6. http://dx.doi.org/ 10.1128/AAC.44.4.1062-1066.2000

28. Pillai SK, Wennersten C, Venkataraman L, Eliopoulos GM, Moellering RC, Karchmer AW. Development of reduced vancomycin susceptibility in methicillin-susceptible Staphylococcus aureus. Clin Infect Dis. 2009;49:1169-74. http://dx.doi.org/ 10.1086/605636

29. Sakoulas G, Eliopoulos GM, Fowler VG Jr, Moellering RC Jr, Novick RP, Lucindo N, et al. Reduced susceptibility of Staphylococcus aureus to vancomycin and platelet microbicidal protein correlates with defective autolysis and loss of accessory gene regulator (agr) function. Antimicrob Agents Chemother. 2005; 49:2687-92. http://dx.doi.org/10.1128/AAC.49.7.2687-2692.2005
30. Peleg AY, Monga D, Pillai S, Mylonakis E, Moellering RC Jr, Eliopoulos GM. Reduced susceptibility to vancomycin influences pathogenicity in Staphylococcus aureus infection. J Infect Dis. 2009;199:532-6. http://dx.doi.org/10.1086/596511

31. Gaupp R, Lei S, Reed JM, Peisker H, Boyle-Vavra S, Bayer AS, et al. Staphylococcus aureus metabolic adaptations during the transition from a daptomycin susceptibility phenotype to a daptomycin nonsusceptibility phenotype. Antimicrob Agents Chemother. 2015;59:4226-38. http://dx.doi.org/10.1128/AAC.00160-15

32. Wang JL, Lai CH, Lin HH, Chen WF, Shih YC, Hung CH. High vancomycin minimum inhibitory concentrations with heteroresistant vancomycin-intermediate Staphylococcus aureus in methicillin-resistant $S$. aureus bacteraemia patients.

Int J Antimicrob Agents. 2013;42:390-4. http://dx.doi.org/10.1016/ j.ijantimicag.2013.07.010

33. Falcón R, Madrid S, Tormo N, Casan C, Albert E, Gimeno C, et al. Intra- and interinstitutional evaluation of an E-test for vancomycin minimum inhibitory concentration measurement in Staphylococcus aureus blood isolates. Clin Infect Dis. 2015;61:1490-2. http://dx.doi.org/10.1093/cid/civ583

34. Rio-Marques L, Hartke A, Bizzini A. The effect of inoculum size on selection of in vitro resistance to vancomycin, daptomycin, and linezolid in methicillin-resistant Staphylococcus aureus. Microb Drug Resist. 2014;20:539-43. http://dx.doi.org/10.1089/ mdr.2014.0059

35. Sharma M, Riederer K, Chase P, Khatib R. High rate of decreasing daptomycin susceptibility during the treatment of persistent Staphylococcus aureus bacteremia. Eur J Clin Microbiol Infect Dis. 2008;27:433-7. http://dx.doi.org/10.1007/s10096-007-0455-5

Address for correspondence: Rafael San-Juan, Unit of Infectious Diseases, Hospital Universitario 12 de Octubre, Instituto de Investigación Hospital 12 de Octubre (i +12$)$, Ave de Córdoba, s/n, 28041 Madrid, Spain; email: rafasjg@yahoo.es

\section{The Public Health Image Library (PHIL)}
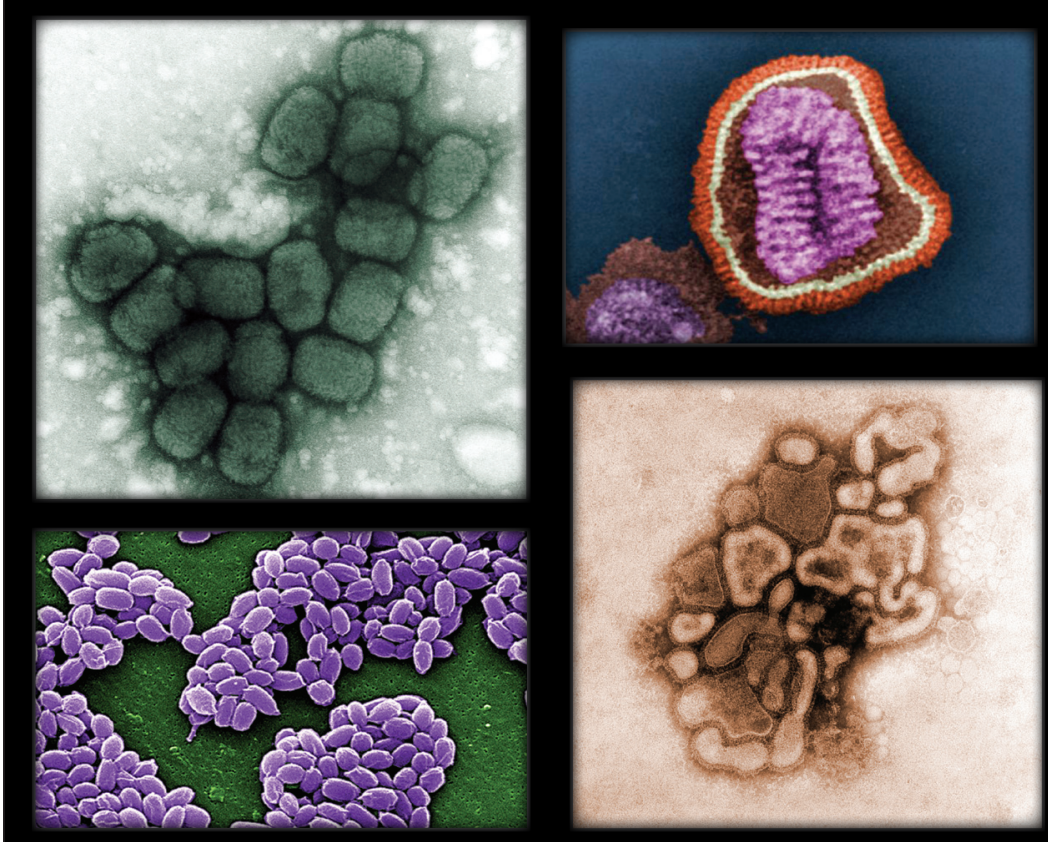

The Public Health Image Library (PHIL), Centers for Disease Control and Prevention, contains thousands of public health-related images, including high-resolution (print quality) photographs, illustrations, and videos.

PHIL collections illustrate current events and articles, supply visual content for health promotion brochures, document the effects of disease, and enhance instructional media.

PHIL Images, accessible to PC and Macintosh users, are in the public domain and available without charge.

$$
\begin{gathered}
\text { Visit PHIL at } \\
\text { http://phil.cdc.gov/phil }
\end{gathered}
$$

